OPEN ACCESS

Check for updates

\section{Continued versus discontinued oxytocin stimulation in the active phase of labour (CONDISOX): double blind randomised controlled trial}

\author{
Sidsel Boie, ${ }^{1}$ Julie Glavind, ${ }^{2}$ Niels Uldbjerg, ${ }^{2}$ Philip J Steer, ${ }^{3}$ Pinar Bor, ${ }^{1}$ on behalf of the \\ CONDISOX trial group
}

${ }^{1}$ Department of Obstetrics and

Gynaecology, Randers Regional Hospital, Randers, Denmark

${ }^{2}$ Department of Obstetrics and Gynaecology, Aarhus University Hospital, Aarhus, Denmark

${ }^{3}$ Academic Department of

Obstetrics and Gynaecology,

Chelsea and Westminster

Hospital, Imperial College

London, London, UK

Correspondence to: S Boie

sidselboie@clin.au.dk

(or @BoieSidsel on Twitter:

Additional material is published

online only. To view please visit the journal online.

Cite this as: $B M J$ 2021;372:n716 http://dx.doi.org/10.1136/bmj.n716

Accepted: 25 February 2021
ORCID 0000-0002-8725-2117)

\section{ABSTRACT}

OBJECTIVE

To determine whether discontinuing oxytocin stimulation in the active phase of induced labour is associated with lower caesarean section rates.

DESIGN

International multicentre, double blind, randomised controlled trial.

SETTING

Nine hospitals in Denmark and one in the Netherlands between 8 April 2016 and 30 June 2020 .

\section{PARTICIPANTS}

1200 women stimulated with intravenous oxytocin infusion during the latent phase of induced labour.

INTERVENTION

Women were randomly assigned to have their oxytocin stimulation discontinued or continued in the active phase of labour.

MAIN OUTCOME MEASURE

Delivery by caesarean section.

RESULTS

A total of 607 women were assigned to discontinuation and 593 to continuation of the oxytocin infusion. The rates of caesarean section were $16.6 \%(n=101)$ in the discontinued group and $14.2 \%$ $(\mathrm{n}=84)$ in the continued group (relative risk $1.17,95 \%$ confidence interval 0.90 to 1.53 ). In 94 parous women with no previous caesarean section, the caesarean section rate was $7.5 \%(11 / 147)$ in the discontinued group and $0.6 \%(1 / 155)$ in the continued group (relative risk $11.6,1.15$ to 88.7 ). Discontinuation was associated with longer duration of labour (median

\section{WHAT IS ALREADY KNOWN ON THIS TOPIC}

Four meta-analyses suggest that once a woman is in active labour, the labour process continues even if oxytocin stimulation is stopped and results in a lower risk of caesarean section

A 2018 Cochrane review questions the quality of previous studies owing to design limitations and judges many of the trials to be at either high or unclear risk of bias across several "risk of bias" domains

\section{WHAT THIS STUDY ADDS}

This is the largest truly double blinded trial on discontinuation of oxytocin stimulation in the active phase of induced labour

In a setting where monitoring of the fetal condition and the uterine contractions can be guaranteed, routine discontinuation of oxytocin stimulation may lead to a small increase in the rate of caesarean section

However, the risks of uterine hyperstimulation and abnormal fetal heart rate were significantly reduced after discontinuation from randomisation to delivery 282 v $201 \mathrm{~min}$; $\mathrm{P}(0.001)$, a reduced risk of hyperstimulation (20/546 (3.7\%) v 70/541 (12.9\%); P<0.001), and a reduced risk of fetal heart rate abnormalities (153/548 (27.9\%) v 219/537 (40.8\%); P<0.001) but rates of other adverse maternal and neonatal outcomes were similar between groups.

\section{CONCLUSIONS}

In a setting where monitoring of the fetal condition and the uterine contractions can be guaranteed, routine discontinuation of oxytocin stimulation may lead to a small increase in caesarean section rate but a significantly reduced risk of uterine hyperstimulation and abnormal fetal heart rate patterns.

TRIAL REGISTRATION

ClinicalTrials.gov NCT02553226.

\section{Introduction}

Approximately a quarter of all term pregnant women have their labour induced. ${ }^{1-3}$ This often includes stimulation with oxytocin. ${ }^{4}$ The stimulation requires a delicate balance between the wish for progression of labour and the risks to the fetus and mother of uterine hyperstimulation, defined as more than five contractions in 10 minutes in response to oxytocin. ${ }^{5-7}$ Different approaches to oxytocin administration have been suggested, such as pulsatile or intermittent administration, ${ }^{8}{ }^{9}$ an automatic feedback system, ${ }^{10}$ high versus low dose, ${ }^{11}$ and/or discontinuation of the stimulation when the active phase of labour is reached. $^{12}$

Four meta-analyses and two more recent studies have suggested that once a woman is in active labour, the labour will continue even if oxytocin stimulation is stopped, resulting in a lower risk of caesarean section for fetal indications secondary to uterine hyperstimulation. ${ }^{12-17}$ However, only one study randomised women at time of the intervention, whereas the remaining studies randomised women in early labour and consequently included a significant fraction of women who delivered by caesarean section before they received the intervention. ${ }^{12}$ In the Cochrane meta-analysis, a subanalysis restricted to trial participants who actually reached the active phase of labour showed little or no effect on the caesarean section rate. ${ }^{12}$ Whether discontinuing oxytocin stimulation is advantageous therefore remains uncertain. The aim of this trial was to test whether discontinuation of oxytocin stimulation, once the active phase of induced labour is achieved, reduces the overall caesarean section rate. 


\section{Methods \\ Trial design}

We conducted a double blind, randomised, placebo controlled multicentre trial. Recruitment took place in nine delivery wards in Denmark and one in the Netherlands. The protocol was published in $2018 .^{18}$

\section{Participants}

Women were eligible if they had a singleton live fetus with a cephalic presentation at term and were stimulated with oxytocin for elective induction of labour or following spontaneous pre-labour rupture of membranes without progression in labour. The exclusion criteria were age under 18 years, need for an interpreter to understand the information material, multiple pregnancy, more than one previous caesarean section, greater than $4 \mathrm{~cm}$ of cervical dilatation at the time of oxytocin infusion, fetal heart rate abnormalities before oxytocin infusion, and an estimated fetal weight of more than $4500 \mathrm{~g}$. All participants gave verbal and written informed consent before randomisation on or after initiation of induction of labour.

\section{Randomisation and masking}

Inclusion and randomisation were primarily facilitated by the midwives on duty. They used an internet based randomisation programme to randomly assign eligible women to one of the two interventions in a one to one ratio. Random block sizes of four, six, and eight were used, and the women were stratified by site, parity (nulliparous; parous with or without previous

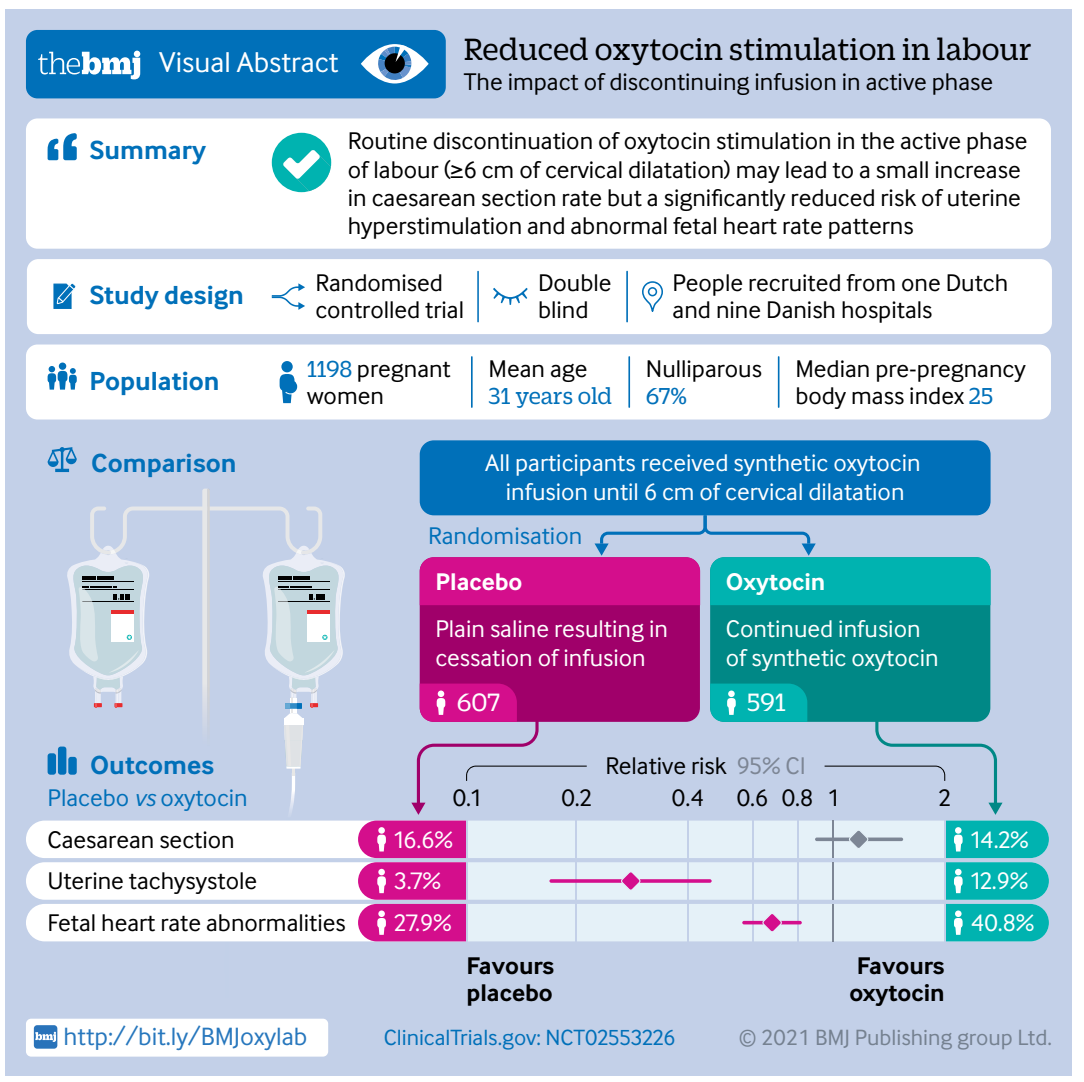

caesarean), and indication for oxytocin infusion (induction of labour or induction due to pre-labour rupture of membranes). The randomisation number generated by the computer program corresponded to the number on the study medication (Denmark: masked, identical ampoules; Netherlands: masked, identical infusions). In case of technical or staff difficulties with the computerised randomisation $(\mathrm{n}=26)$, the women were given the study medication with the lowest available number. Accordingly, women, care givers, and trial managers were all blinded to the allocation.

\section{Oxytocin stimulation protocol}

The stimulation regimen used in this study was similar to the current Danish and Dutch recommendations on oxytocin stimulation for induced labour. An intravenous infusion of 10 IU oxytocin (Sigma Tau Pharmaceuticals) diluted in $1000 \mathrm{~mL}$ of isotonic saline (Denmark) or 5 IU oxytocin diluted in $50 \mathrm{~mL}$ of isotonic saline (Netherlands) was started at $3.3 \mathrm{mIU} / \mathrm{min}$ and increased every 20 minutes by $3.3 \mathrm{mIU} / \mathrm{min}$ until regular contractions (three to five contractions every 10 minutes) were achieved. The maximum authorised infusion rates were $30 \mathrm{mIU} / \mathrm{min}$ in Denmark and $33 \mathrm{mIU} /$ minute in the Netherlands. Women were randomised when the active phase of labour was established, defined as ruptured membranes with complete effacement of the cervix, cervical dilatation of at least $6 \mathrm{~cm}$, and at least three contractions every 10 minutes. After randomisation, the infusion was replaced by the study medication. The continued group received oxytocin at the standard concentration, whereas the discontinued group received placebo with saline. The study medication was set to the same infusion rate as the initial oxytocin infusion and was adjusted (discontinued, reduced, or increased) by the birth attendants according to the clinical situation. All participants were continuously monitored with cardiotocography. Antibiotics were recommended if the woman was a candidate for group B Streptococcus prophylaxis, in case of an isolated temperature of $38.5^{\circ} \mathrm{C}$ or higher, or in case of maternal pyrexia during labour (temperature $\geq 38.2^{\circ} \mathrm{C}$ with epidural; $\geq 38^{\circ} \mathrm{C}$ without epidural) combined with signs of intrauterine infection or combined with an interval since rupture of membranes exceeding 18 hours.

We defined slow progress (dystocia) in the first stage of labour as a cervical dilatation of less than $2 \mathrm{~cm}$ every four hours despite three to five uterine contractions in 10 minutes and/or after reaching the maximum infusion dose. We defined slow labour progress in the passive second stage as descent of the presenting part not reaching $2 \mathrm{~cm}$ below the ischial spines within three hours and in the active second stage if delivery was not achieved within two hours of maternal expulsive efforts. In women who fulfilled these dystocia criteria, the study medication was replaced with open label administration of oxytocin infusion with a starting dose rate of $3.3 \mathrm{mIU} / \mathrm{min}$. 


\section{Trial outcomes}

The primary outcome was caesarean section. Secondary maternal and neonatal outcomes are listed in the tables of results. Data collection was carried out by the researcher (midwife or physician) at each participating site and managed using an electronic data capturing tool hosted at Aarhus University, REDCap (Research Electronic Data Capture). ${ }^{21}$ The Childbirth Experience Questionnaire was sent to the women one month after the birth to evaluate the mothers' expectations and childbirth experience. The transcultural adapted Danish Childbirth Experience Questionnaire assesses three domains of the experience of childbirth: the woman's own capacity (her sense of control and personal feelings during childbirth), professional support (the woman's receipt of information and midwifery care), and participation in the birth (the woman's ability to influence the birthing process). ${ }^{22}$ Responses were scored according to the scoring instructions of the Danish questionnaire. ${ }^{22}$ Scores in each domain range from 1 to 4, with higher scores indicating a better childbirth experience.

\section{Statistical analysis}

The power calculation was based on data from an earlier Danish study in which the caesarean section rate in women continuously stimulated with oxytocin was $22 \%$, compared with $15 \%$ in the discontinued group. ${ }^{23}$ On the basis of these numbers and with an $\alpha$ of 0.05 , a total sample size of 1200 women (600 per group) would provide a power of at least $80 \%$ to detect a difference of $7 \%$ in the caesarean section rate between the two regimens.

We analysed participants according to intention to treat in their assigned group, regardless of their adherence to the assignment. Baseline demographic data were presented with counts and percentages for categorical variables, means and standard deviations for continuous Gaussian distributed variables, and medians and interquartile ranges for continuous nonGaussian variables. We assessed the primary outcome variable by using a $\chi^{2}$ square test with a significance threshold $\mathrm{P}$ value of below 0.05 to compare the event rates in the two groups. We presented the results as absolute and relative risks along with 95\% confidence intervals. We assessed categorical secondary outcomes in the same way as the primary outcome. For continuous secondary outcomes with a Gaussian distribution (after log transformation if appropriate), we assessed differences between groups by using the student's $t$ test; if the data were non-Gaussian, we used a non-parametric Mann-Whitney U test. Non-inferiority testing was performed with a pre-stated margin of noninferiority boundary at 1.09 .

To assess the women's experience of childbirth as measured with the Childbirth Experience Questionnaire, we did a complete case analysis, using the unpaired $t$ test, to compare the mean subscale scores and the mean total score (the average of the individual subscale scores) between women in the two groups. We used a Mann-Whitney U test to calculate P values. In cases with a few missing items, we used the half scale method so that when the respondent had answered at least half the items in the scale, the sum of the scores was divided by the number of answered items. ${ }^{24}$

For the primary outcome, we did pre-specified subgroup analyses according to parity (nulliparous, parous, and parous with previous caesarean section) and indication for stimulation (pre-labour rupture of membranes or induction of labour). We used Stata software, version 14, for all analyses.

\section{Trial monitoring}

Assessment for safety and adverse events was based on the most recent available summary of product characteristics for oxytocin. An independent Data Monitoring and Ethics Committeemet yearly throughout the trial to discuss the yearly interim analyses conducted during the course of the trial for the purpose of monitoring efficacy and safety. This committee elaborated a yearly report for the independent Trial Steering Committee, with a conclusion on trial safety and progression based on the interim analysis. The Trial Steering Committee provided independent advice to the trialists based on the Data Monitoring and Ethics Committee's conclusion.

\section{Patient and public involvement}

As it was not customary in Denmark when the trial was designed, no women were involved in setting the research question or the outcome measures, nor were they involved in developing plans for recruitment, design, or implementation of the study, and no women were asked to advise on interpretation or writing up of results.

\section{Results}

A total of 1200 women were randomised between 8 April 2016 and 30 June 2020 (fig 1). Two women in the continued group subsequently withdrew consent, and their data were therefore not included in the final analysis. The number of women recruited from each site is shown in supplementary table A.

A total of 1849 women signed the informed consent form, of whom 649 (35\%) were not randomised; in 202 (11\%) women, this was due to rapid progression of labour (supplementary table B). For reasons shown in figure 1, $127(11 \%)$ women never received the allocated intervention ( 65 women in the discontinued group and 62 women in the continued group). The baseline characteristics were similar in the two groups (table 1).

Data on the primary outcome were available for all women included in the intention to treat analysis. No statistically significant difference existed in the frequency of caesarean section between the discontinued group (101 (16.6\%) caesarean sections in 607 women) and the continued group (84 (14.2\%) caesarean sections in 591 women) (relative risk $1.17,95 \%$ confidence interval 0.90 to 1.53 ; absolute difference $2.4 \%,-1.7 \%$ to $6.5 \%$ ). The pre-specified non-inferiority testing (with a boundary of 1.09) was 


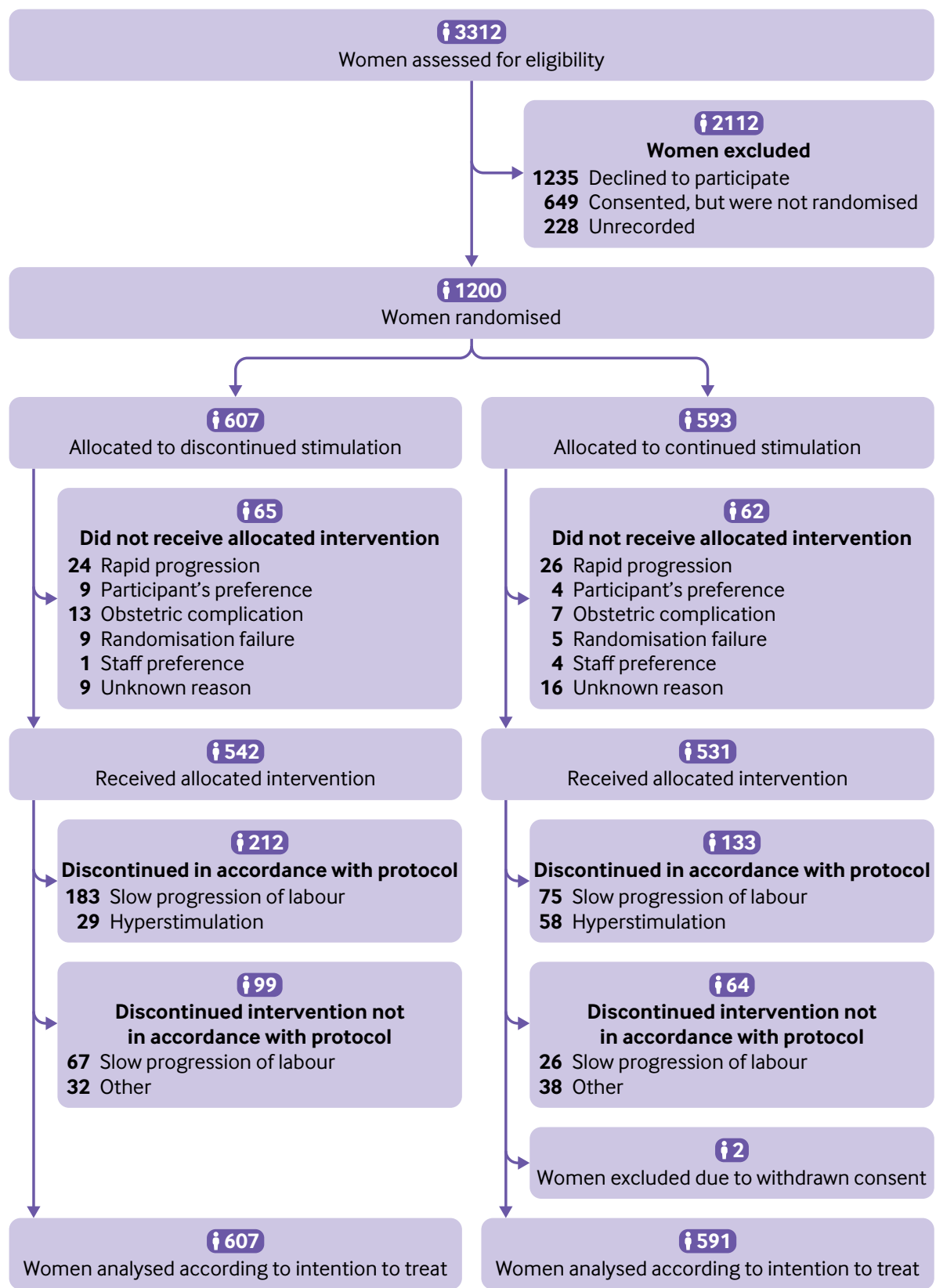

Fig 1 | Consort flow diagram

inconclusive $(\mathrm{P}=0.70)$. The result was not substantially different in a per protocol analysis (relative risk 1.30, 0.94 to 1.80 ).

Data on secondary outcomes showed that discontinuation of oxytocin was associated with a longer duration of labour (median from randomisation to delivery $282 v 201 \mathrm{~min} ; \mathrm{P}<0.001)$, a reduced risk of hyperstimulation $(3.7 \% v 12.9 \%$; $\mathrm{P}<0.001)$, a reduced risk of fetal heart rate abnormalities $(27.9 \% v 40.8 \%$; $\mathrm{P}<0.001$ ), and similar rates of other adverse maternal and neonatal outcomes (table 2 and table 3). Among the 858 (72\%) women who returned the Childbirth Experience Questionnaire four weeks postpartum, we observed no significant differences between the two groups in the subscale scores or total scores (indicating level of satisfaction with the childbirth experience) (table 4).

The pre-specified subgroup analysis in 94 parous women with no previous caesarean section found a caesarean section rate of $7.5 \%(11 / 147)$ in the discontinued group and $0.6 \%(1 / 155)$ in the continued group (relative risk $11.6,1.15$ to 88.7 ). The other prespecified subgroup analyses did not find any significant difference in caesarean section rate between groups. In 802 nulliparous women, the caesarean section rate was $18.8 \%$ for both groups (76/404 in the discontinued group; 75/398 in the continued group) (relative risk $1.00,0.75$ to 1.33$)$. In parous women with previous caesarean section, the caesarean section rate was $25 \%(14 / 56)$ in the discontinued group versus $21.1 \%$ 


\begin{tabular}{|c|c|c|}
\hline \multirow[b]{2}{*}{ Baseline characteristics } & \multicolumn{2}{|c|}{ Study population } \\
\hline & Discontinued $(n=607)$ & Continued $(n=591)$ \\
\hline Mean (SD) maternal age, years & $31.0(4.9)$ & $31.3(4.9)$ \\
\hline White European & $531 / 583(91.1)$ & $510 / 561(90.9)$ \\
\hline Median (IQR) pre-pregnancy BMI & $25.2(22.2-29.8)$ & $24.8(22.1-29.0)$ \\
\hline Pre-pregnancy BMI >30 & $151(24.9)$ & $126(21.3)$ \\
\hline Smoking during pregnancy & $96 / 593(16.2)$ & $79 / 580(13.6)$ \\
\hline Married or living with partner & $538 / 597(90.1)$ & $534 / 586(91.1)$ \\
\hline \multicolumn{3}{|l|}{ Parity: } \\
\hline Nulliparous & $404(66.6)$ & $398(67.3)$ \\
\hline Parous with no previous caesarean section & $147(24.2)$ & $155(26.2)$ \\
\hline Parous with previous caesarean section & $56(9.2)$ & $38(6.4)$ \\
\hline \multicolumn{3}{|l|}{ Medical history during pregnancy: } \\
\hline Gestational diabetes & $53(8.7)$ & $58(9.8)$ \\
\hline Diabetes mellitus type 1 & $8(1.3)$ & $5(0.8)$ \\
\hline Diabetes mellitus type 2 & $7(1.2)$ & $1(0.2)$ \\
\hline Hypertensive disorders* & $97(16.0)$ & $92(15.6)$ \\
\hline Small for gestational age $\mathrm{e}^{19}$ & $13(2.1)$ & $15(2.5)$ \\
\hline Autoimmune/Inflammatory disease $\dagger$ & $31(5.1)$ & $35(5.9)$ \\
\hline Use of antidepressive medication at term & $21(3.5)$ & $18(3.0)$ \\
\hline Median (IQR) length of gestation at birth, weeks & $40^{+3}\left(39^{+1}-41^{+2}\right)$ & $40^{+3}\left(39^{+1}-41^{+3}\right)$ \\
\hline \multicolumn{3}{|l|}{ Indication for labour induction: } \\
\hline Ruptured membranes & $230(37.9)$ & $221(37.4)$ \\
\hline Diabetes & $43(7.1)$ & $39(6.6)$ \\
\hline Postdate pregnancy $\ddagger$ & $102(16.8)$ & $120(20.3)$ \\
\hline Hypertensive disorders* & $66(10.9)$ & $71(12.0)$ \\
\hline $\mathrm{BMI} \geq 35 \S$ & $24(4.0)$ & $18(3.0)$ \\
\hline Oligohydramniosף & $14(2.3)$ & $8(1.4)$ \\
\hline Maternal request & $27(4.4)$ & $21(3.6)$ \\
\hline Other ${ }^{\star \star}$ & $101(16.6)$ & $93(15.7)$ \\
\hline \multicolumn{3}{|l|}{ Cervical ripening: } \\
\hline Prostaglandins & $253(41.7)$ & $237(40.1)$ \\
\hline Cervical ripening catheter & $84(13.8)$ & $85(14.4)$ \\
\hline Both & $39(6.4)$ & $40(6.8)$ \\
\hline Cervical dilatation at time of randomisation: & $(n=590)$ & $(n=565)$ \\
\hline$<6 \mathrm{~cm}$ & $15(2.5)$ & $7(1.2)$ \\
\hline $6 \mathrm{~cm}$ & $256(42.2)$ & $272(46.0)$ \\
\hline $7 \mathrm{~cm}$ & $108(17.8)$ & $118(20.0)$ \\
\hline $8 \mathrm{~cm}$ & $96(15.8)$ & $71(12.0)$ \\
\hline $9 \mathrm{~cm}$ & $52(8.6)$ & $33(5.6)$ \\
\hline $10 \mathrm{~cm}$ & $63(10.4)$ & $64(10.8)$ \\
\hline Mean (SD) birth weight, $\mathrm{g}$ & $3646(509)$ & $3596(502)$ \\
\hline Female sex of newborn & $283(46.6)$ & $275(46.6)$ \\
\hline \multicolumn{3}{|c|}{ 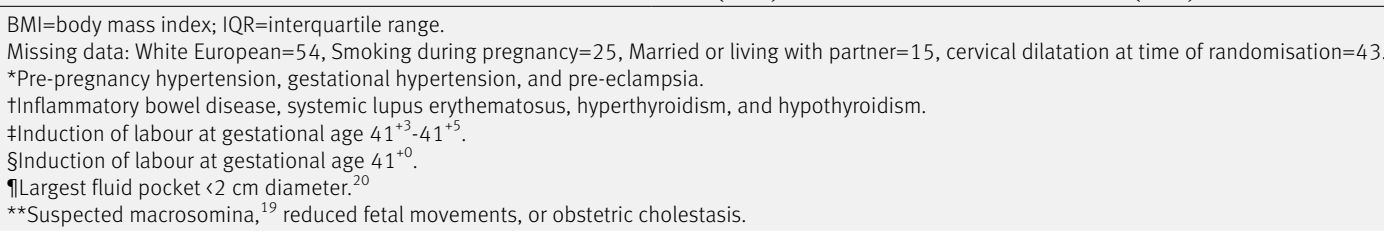 } \\
\hline
\end{tabular}

$(8 / 38)$ in the continued group (relative risk 1.19, 0.55 to 2.55$)$. In the 779 women with induced labour starting with intact membranes, $17.5 \%$ (70/400) in the discontinued group had a caesarean section compared with $16.9 \%$ (64/379) in the continued group (relative risk $1.04,0.76$ to 1.41$)$. In the 419 women with prelabour rupture of membranes, 15\% (31/207) in the discontinued group delivered by caesarean section compared with $9.5 \%(20 / 212)$ in the continued group (relative risk 1.58, 0.94 to 2.69).

Stopping the study medication happened both in accordance and not in accordance with the protocol for the reasons listed in table 5 . The total number of women who stopped the study medication differed between the two groups: $311(51.2 \%)$ in the discontinued group and $197(33.3 \%)$ in the continued group $(\mathrm{P}<0.0001)$. In cases of slow labour progress as the reason for stopping, all women had study medication changed to open label oxytocin.

Because of the large number of women in whom the trial medication was stopped not in accordance with the protocol, we did a further analysis by actual treatment. We compared the women who had placebo throughout $(n=224)$ or oxytocin throughout $(n=328)$ with those switched to open label oxytocin (311 originally allocated to discontinued treatment and 197 originally allocated to continued treatment). Women in the groups continuing with study medication throughout had very low caesarean section rates compared with women who were switched to open label 
oxytocin (13/224 (5.8\%) and $17 / 328(5.2 \%)$ versus $73 / 311$ (23.5\%) and 57/197 (28.9\%); $\mathrm{P}<0.0001)$ and were more likely to be parous $(104 / 224(46.4 \%)$ and $119 / 328(36.3 \%)$ versus $64 / 311(20.6 \%)$ and $40 / 197$ (20.3\%); $\mathrm{P}<0.0001)$. In addition, women not switched to open label oxytocin had smaller babies (mean 3586 (SD 528) g and 3582 (492) g versus 3697 (499) g and 3665 (504) g) and shorter labours (mean 404 (244) min and 461 (313) min versus 747 (322) min and 731 (347) min; $\mathrm{P}<0.0001$ ).

One neonatal death was reported in the discontinued group. The macrosomic baby was born vaginally of a diabetic mother, with an Apgar score of $1 / 1$ and $7 / 5$. The baby was immediately resuscitated by intubation and was then admitted to the neonatal intensive care unit. The baby was diagnosed as having severe haemolysis and a subgaleal haematoma and died aged 5 days.

The randomisation code was broken before delivery in two women owing to withdrawal of consent and on maternal request to change to open label administration if not already given. In one woman, the randomisation code was broken three months postpartum on maternal request in a participant with

\begin{tabular}{|c|c|c|c|c|}
\hline \multirow[b]{2}{*}{ Maternal outcomes } & \multicolumn{2}{|c|}{ Study population } & \multirow[b]{2}{*}{ Relative risk $(95 \% \mathrm{Cl})$} & \multirow[b]{2}{*}{$P$ value } \\
\hline & Discontinued $(n=607)$ & Continued $(n=591)$ & & \\
\hline \multicolumn{5}{|l|}{ Primary outcome } \\
\hline Caesarean section & $101(16.6)$ & $84(14.2)$ & $1.17(0.90$ to 1.53$)$ & 0.25 \\
\hline \multicolumn{5}{|l|}{ Secondary maternal outcomes } \\
\hline Indication for caesarean section*: & & & - & - \\
\hline Dystocia & $62(10.2)$ & $58(9.8)$ & & \\
\hline Suspicion of fetal asphyxia & $26(4.3)$ & $13(2.2)$ & - & - \\
\hline Suspicion of uterine rupture & $3(0.5)$ & $1(0.2)$ & - & - \\
\hline Chorioamnionitis & $2(0.3)$ & $1(0.2)$ & - & - \\
\hline Other & $8(1.3)$ & $11(1.9)$ & - & - \\
\hline Assisted vaginal delivery (use of forceps or vacuum) & $64(10.5) \dagger$ & $67(11.3) \dagger$ & $0.93(0.67$ to 1.28$)$ & 0.66 \\
\hline $\begin{array}{l}\text { Median (IQR) duration from start of initial } \\
\text { oxytocin infusion to delivery, min }\end{array}$ & $535(314-797)$ & $477(272-727)$ & - & 0.01 \\
\hline $\begin{array}{l}\text { Median (IQR) duration from time of } \\
\text { randomisation to delivery, min }\end{array}$ & $282(119-484)$ & $203(77-397)$ & - & $<0.001$ \\
\hline Median (IQR) duration of second stage of labour $\star$, min & $95(31-198)$ & $78(29-182)$ & - & 0.07 \\
\hline Epidural use: & $386(63.6)$ & $350(59.2)$ & $1.07(0.98$ to 1.17$)$ & 0.14 \\
\hline Before randomisation & $304(50.1)$ & $295(49.9)$ & $1.00(0.90$ to 1.12$)$ & 0.95 \\
\hline After randomisation & $83(13.7)$ & $57(9.6)$ & $1.42(1.03$ to 1.95$)$ & 0.03 \\
\hline \multicolumn{5}{|l|}{ Uterine tachysystole during labour: } \\
\hline Before intervention & $61 / 599(10.2)$ & $80 / 585(13.7)$ & $0.74(0.54$ to 1.02$)$ & 0.06 \\
\hline During intervention & $20 / 546(3.7)$ & 70/541 (12.9) & $0.28(0.17$ to 0.46$)$ & $<0.001$ \\
\hline Third or fourth degree perineal tears§ & $25 / 506(4.9)$ & $27 / 507(5.3)$ & $0.92(0.54$ to 1.57$)$ & 0.76 \\
\hline Postpartum haemorrhage within $24 \mathrm{~h}$ : & $(n=605)$ & $(n=590)$ & & \\
\hline$>1000 \mathrm{~mL}$ & $84(13.8)$ & $102(17.3)$ & 0.80 (0.61 to 1.05$)$ & 0.10 \\
\hline$>1500 \mathrm{~mL}$ & $43(7.1)$ & $51(8.6)$ & $0.82(0.56$ to 1.21$)$ & 0.32 \\
\hline Postpartum blood transfusion & $8(13.2)$ & $11(18.6)$ & $0.71(0.29$ to 1.75$)$ & 0.45 \\
\hline Evacuation of retained tissue & $39 / 605(7.7)$ & 45/590(8.9) & $0.86(0.58$ to 1.31$)$ & 0.50 \\
\hline Maternal pyrexia during labour* & $70 / 606(11.6)$ & $52 / 591(8.8)$ & $1.31(0.93$ to 1.85$)$ & 0.12 \\
\hline Uterine rupture & 0 & 0 & .. & .. \\
\hline Urinary retention & $25 / 601(4.2)$ & $20 / 586(3.4)$ & $1.22(0.68$ to 2.17$)$ & 0.50 \\
\hline Puerperal infection during admission ${ }^{\star \star}$ & $26 / 601(4.3)$ & $27 / 591(4.6)$ & $0.95(0.56$ to 1.60$)$ & 0.83 \\
\hline \multicolumn{5}{|l|}{ Postpartum hospital stay: } \\
\hline$\leq 2$ days & $261(43.0)$ & $284(48.1)$ & $0.89(0.79$ to 1.10$)$ & 0.08 \\
\hline$>2$ days & $346(57.0)$ & $307(51.9)$ & $1.10(0.99$ to 1.22$)$ & 0.08 \\
\hline Exclusive breast feeding at discharge ${ }^{\star}$ & 403/589 (68.4) & $378 / 569(66.4)$ & $1.03(0.95$ to 1.12$)$ & 0.47 \\
\hline Readmission within 7 days: & $31 / 605(5.1)$ & $41 / 589(7.0)$ & $0.74(0.47$ to 1.16$)$ & 0.18 \\
\hline Puerperal infectiont† & $11(1.8)$ & $9(1.5)$ & - & - \\
\hline Bowel obstruction & $1(0.2)$ & $2(0.4)$ & - & - \\
\hline Thrombo-embolic complication & 0 & $1(0.2)$ & - & - \\
\hline Otherł‡ & $19(3.1)$ & $29(4.9)$ & - & - \\
\hline Maternal death within 7 days & 0 & 0 & - & - \\
\hline \multicolumn{5}{|c|}{ 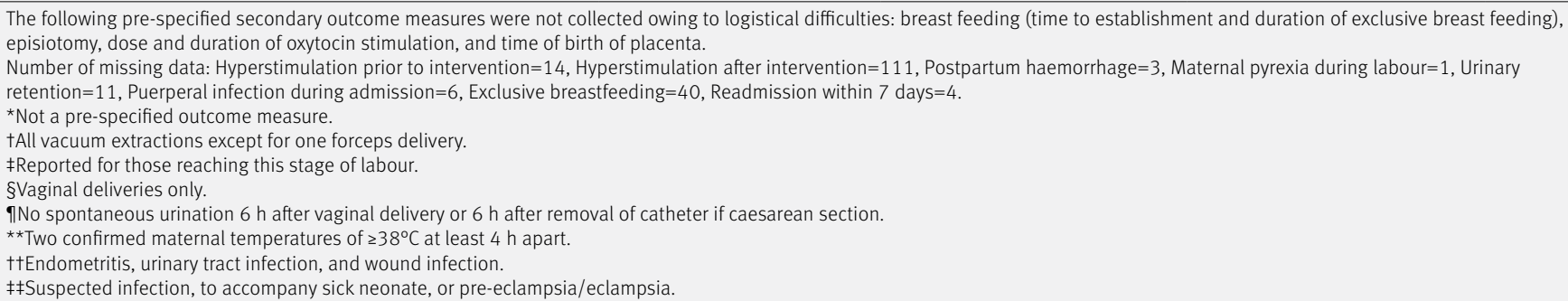 } \\
\hline
\end{tabular}


postnatal depression; at this point all relevant data had already been entered in the database.

\section{Discussion}

This double blind, randomised multicentre trial found that, in a setting where monitoring of the fetal condition and the uterine contractions can be guaranteed, a policy of discontinuation of oxytocin may lead to a small increase in the risk of caesarean section. On the other hand, the risk of uterine hyperstimulation and abnormal fetal heart rate was significantly reduced when oxytocin was discontinued, and this may be important in settings where close observation of mother and fetus may be challenging owing to shortages of resources. Other maternal and neonatal outcomes, including the women's birth experience, did not differ significantly between the groups.

\section{Strengths and limitations of study}

The strength of our study is the well organised, multicentred, and truly double blind design and the large sample size. The blinding reduces or eliminates the experimental biases that may arise from the expectations of the women and the expectations of the birth attendants, whose own expectations may have an important effect on the clinical management of labour. Furthermore, randomisation and intervention were concomitant, resulting in only two post-randomisation dropouts (due to withdrawn consent) compared with $5.0-13.2 \%$ dropouts in previous trials. ${ }^{12}$
A significant limitation of the trial was the relatively high proportion of women who stopped the study medication both in accordance with the protocol $(212 / 607(34.9 \%)$ in the discontinued group and $133 / 591(22.5 \%)$ in the continued group) and not in accordance with the protocol (99/607 (16.3\%) in the discontinued group and 64/591 (10.8\%) in the continued group). Previous open label trials have reported similar proportions of women (36-46.4\%) in the discontinued group having oxytocin restarted. ${ }^{23} 26$

For reasons not recorded because the phenomenon was not anticipated, if the birth attendants decided to restart stimulating the labour, they usually chose to use open label oxytocin rather than the study medication. This resulted in some loss of power with regard to our primary hypothesis, so a reduction in the caesarean section rate from discontinuation of the oxytocin has not been ruled out (the lower confidence interval is a $1.7 \%$ reduction in caesarean section rate).

Anecdotally, birth attendants involved with the trial reported a general impatience with slower than average rates of cervical dilatation, leading to pressure from medical and midwifery colleagues to restart oxytocin even though the trial criteria for this had not been met. Ambiguity about oxytocin use among birth attendants has been reported previously. ${ }^{27}$ Furthermore, the high number of women being changed to open label infusion could also reflect insecurity among the birth attendants as to whether placebo is a safe option. This could be an argument for repeating the trial, as

\begin{tabular}{|c|c|c|c|c|}
\hline \multirow[b]{2}{*}{ Neonatal outcomes } & \multicolumn{2}{|c|}{ Study population } & \multirow[b]{2}{*}{ Relative risk $(95 \% \mathrm{Cl})$} & \multirow[b]{2}{*}{$P$ value } \\
\hline & Discontinued $(n=607)$ & Continued $(n=591)$ & & \\
\hline \multicolumn{5}{|l|}{ Perinatal death: } \\
\hline Stillbirth & 0 & 0 & - & - \\
\hline Within 7 days postpartum & $1(0.2)$ & 0 & - & - \\
\hline \multicolumn{5}{|l|}{ Fetal heart rate abnormalities during labour*: } \\
\hline Before intervention & $103 / 603(17.1)$ & $90 / 584(15.4)$ & $1.11(0.86$ to 1.44$)$ & 0.44 \\
\hline During intervention & $153 / 548(27.9)$ & $219 / 537(40.8)$ & $0.68(0.57$ to 0.81$)$ & $<0.001$ \\
\hline \multicolumn{5}{|l|}{ Apgar score at 5 min: } \\
\hline$<4$ & 0 & 0 & - & - \\
\hline 4-7 & $6(1.0)$ & $4(0.7)$ & $1.46(0.45$ to 5.24$)$ & 0.55 \\
\hline Umbilical cord arterial pH: & $(n=536)$ & $(n=506)$ & & \\
\hline$<7.00$ & $3(0.5)$ & 0 & - & - \\
\hline $7.00-7.10$ & $39(6.4)$ & $40(6.8)$ & $0.95(0.62$ to 1.45$)$ & 0.81 \\
\hline CPAP treatment $24 \mathrm{~h}$ postpartum & $84 / 607$ (13.8) & $72 / 58912.2)$ & $1.13(0.84$ to 1.52$)$ & 0.41 \\
\hline Intubation 24 h postpartum & $1(0.2)$ & $1(0.2)$ & $0.97(0.61$ to 15.5$)$ & 0.98 \\
\hline NICU admission & $53(8.7)$ & $52(8.8)$ & $0.99(0.69$ to 1.43$)$ & 0.96 \\
\hline \multicolumn{5}{|l|}{ Diagnosis during NICU admission: } \\
\hline Respiratory distress & $19(3.1)$ & $21(3.6)$ & - & - \\
\hline Infection & $10(1.6)$ & $6(1.0)$ & - & - \\
\hline Neonatal asphyxia & $1(0.2)$ & $1(0.2)$ & - & - \\
\hline Neonatal seizure & $1(0.2)$ & $1(0.2)$ & - & - \\
\hline Birth traumat & $1(0.2)$ & $2(0.3)$ & - & - \\
\hline Hyperbilirubinaemia & $2(0.3)$ & $2(0.3)$ & - & - \\
\hline Hypoglycaemia & $4(0.7)$ & 0 & - & - \\
\hline Otherł & $12(2.0)$ & $14(2.4)$ & - & - \\
\hline Unknown & $3(0.5)$ & $5(0.8)$ & - & - \\
\hline Treated for hyperbilirubinemia during admission & $31(5.1)$ & $31(5.2)$ & $0.98(0.60$ to 1.59$)$ & 0.94 \\
\hline \multicolumn{5}{|c|}{$\begin{array}{l}\text { CPAP }=\text { continuous positive airway pressure; } \mathrm{NICU}=\text { neonatal intensive care unit. } \\
\text { Number of missing data: Fetal heart rate abnormalities prior to intervention=11, Fetal heart rate abnormalities during intervention=113, umbilical cord arterial } \mathrm{pH}=156, \mathrm{CPAP} \text { treatment=2, } \\
\text { treated for hyperbilirubinemia }=23 \text {. } \\
{ }^{*} \text { Classification by FIGO } 1985 .{ }^{25} \\
+ \text { Cephalohaematoma and/or subgaleal haematoma. } \\
\text { fLow birth weight, congenital malformations, dehydration, or suspected infection. }\end{array}$} \\
\hline
\end{tabular}




\begin{tabular}{|c|c|c|c|}
\hline \multirow[b]{2}{*}{ Domain } & \multicolumn{2}{|c|}{ Study population } & \multirow[b]{2}{*}{$P$ value } \\
\hline & Discontinued $(n=451 ; 75 \%)$ & Continued $(n=407 ; 69 \%)$ & \\
\hline Own capacity & $2.79(0.63)$ & $2.84(0.61)$ & 0.21 \\
\hline Participation & $3.16(0.76)$ & $3.10(0.79)$ & 0.34 \\
\hline Professional support & $3.79(0.40)$ & $3.76(0.42)$ & 0.26 \\
\hline Overall score & $3.11(0.47)$ & $3.13(0.50)$ & 0.66 \\
\hline
\end{tabular}

${ }^{*}$ Mean (SD) Childbirth Experience Questionnaire scores on scale from 1 to 4 , with 4 being optimum.

the results show that discontinuing oxytocin in the active phase of labour is not harmful, and this might encourage birth attendants to stick to the protocol in future.

With respect to external validity, the trial included women from secondary and tertiary level hospitals, and the results are generalisable to countries with similar demographics. Considerable heterogeneity exists in the methods used for induction around the world, ${ }^{428}$ and the methods of induction have differing efficacy. Most participating units in the study used oral prostaglandin ripening followed by amniotomy and oxytocin infusion. However, for parous women with a previous caesarean delivery, cervical priming with a double balloon catheter was preferred. We did not predefine the indications for induction of labour. Hence, whether the results of this trial would be generalisable to centres that use other methods of induction or other oxytocin stimulation regimens is unclear.

During the study period, an additional 202 Danish women consented to participate, but staff were left with no time for randomisation and intervention owing to rapid progression of labour. For the Dutch women $(n=122)$ who consented but were not randomised, the reason was not recorded, but we have no reason to believe that the reasons in the Dutch setting were different. The women with rapid progression of labour might potentially have benefitted the most from discontinuation of oxytocin, and this might explain the lack of difference in the caesarean section rates between the two groups in our study. Discontinuing oxytocin infusion selectively in women with faster than average cervical dilatation rates may thus be an advantage. Future studies could focus on this subgroup.

\section{Comparison with other studies}

The result of the trial differs from those of previously published studies and meta-analyses, which suggested that oxytocin discontinuation significantly reduces the

\begin{tabular}{|c|c|c|c|}
\hline \multirow[b]{2}{*}{ Reason for discontinuation of study medication } & \multicolumn{3}{|c|}{ Study population } \\
\hline & $\begin{array}{l}\text { Discontinued } \\
(n=607)\end{array}$ & $\begin{array}{l}\text { Continued } \\
(n=591)\end{array}$ & $P$ value \\
\hline Slow labour progress in accordance with protocol & $183(30.1)$ & $75(12.7)$ & $<0.001$ \\
\hline Slow labour progress not in accordance with protocol & $67(11.0)$ & $26(4.4)$ & $<0.001$ \\
\hline Uterine tachysystole or FHR abnormalities & $29(4.8)$ & $58(9.8)$ & 0.002 \\
\hline $\begin{array}{l}\text { Other (pyrexia, meconium stained amniotic fluid) } \\
\text { not in accordance with protocol }\end{array}$ & $32(5.3)$ & $38(6.4)$ & 0.42 \\
\hline Total & $311(51.2)$ & $197(33.3)$ & $<0.001$ \\
\hline
\end{tabular}

risk of caesarean section. ${ }^{12-17}$ However, most of the previous studies were designed to measure another primary outcome-namely, duration of labour. Many of these studies had limitations: imbalanced postrandomisation dropouts before intervention, open label administration, and small sample sizes of 100200 women.

Furthermore, our population differs in terms of cervical dilatation at the time of intervention, inclusion of women with previous caesarean section, proportion of nulliparous women, and birth weight. ${ }^{12}$ In contrast to our findings on caesarean section rate, we agree with all other published papers that the rate of uterine hyperstimulation and abnormal fetal heart rate is significantly and substantially lower when oxytocin stimulation is discontinued in the active phase of induced labour. Although we saw no significant effect on neonatal outcome in our closely monitored environment, this may be of considerable importance in settings where close monitoring of the mother and baby is not feasible.

Also, in line with several previous studies, ${ }^{12}{ }^{29}$ we found oxytocin stimulation to have little or no effect on the duration of the second stage of labour. This suggests that oxytocin might not be necessary during descent and expulsion of the fetus.

\section{Conclusion}

We conclude that in a setting where monitoring of the fetal condition and the uterine contractions can be guaranteed, routine discontinuation of oxytocin stimulation may lead to a small increased risk of caesarean section but a significantly reduced risk of uterine hyperstimulation and abnormal fetal heart rate.

We thank all the families who participated in this trial and the dedicated staff from all participating departments. Furthermore, we acknowledge the valuable contributions from each member of the Trial Steering Committee (Jim Thornton (chair), Thomas Bergholt, Wessel Ganzevoort, and Jens Fuglsang) and each member of the Data Monitoring and Ethics Committee (Lone Krebs (chair), Gorm Greisen, and Martin Johansen).

Members of the CONDISOX trial group: Attila Bothazi, consultant in obstetrics and gynaecology, Aalborg University Hospital, Aalborg, Denmark; Iben Sundtoft, senior consultant in obstetrics, Herning Regional Hospital, Herning, Denmark; Jannet Bakker, research midwife, Amsterdam UMC, Amsterdam University Hospital, Amsterdam, Netherlands; Joris Van der Post, professor in obstetrics, Amsterdam UMC, Amsterdam University Hospital, Amsterdam, Netherlands; Kristina Martha Renault, senior consultant in obstetrics, Juliane Marie Centre, Copenhagen University Hospital, Rigshospitalet, Copenhagen, Denmark; Lene Drasbek Huusom, senior consultant in obstetrics, Copenhagen University Hospital, Hvidovre Hospital, Hvidovre, Denmark; Lone Hvidman, senior consultant in obstetrics, Aarhus University Hospital, Aarhus, Denmark; Maja Thode Rask, consultant in obstetrics, Odense University Hospital, Odense, Denmark: Mohammed Khalil, senior consultant in obstetrics, Lillebaelt Hospital, Kolding, Denmark; Nini Møller; senior consultant in obstetrics, Copenhagen University Hospital, Nordsjaelland Hospital, Hilleroed, Denmark; Tine Greve, consultant in obstetrics, Copenhagen University Hospital, Hvidovre Hospital, Hvidovre, Denmark.

Contributors: SB, PB, JG, NU, and PS conceived and designed the study. The members of the Trial Steering Committee and Data Monitoring and Ethics Committee contributed to the design. SB, PB $J G, N U, J B, A B, L H, I S, M K, M R, K R, L D H, T G$, and NM conducted the study. Representatives of each centre entered the data, and the data was cross checked by good clinical practices monitors at all centres. SB did the statistical analysis, with overview by an external statistician and PS. SB, PB, JG, NU, and PS interpreted the data. SB drafted the 
original manuscript, with major contributions from PB, JG, NU, and PS. All authors reviewed and revised the manuscript. All authors read and approved the final manuscript. All authors have agreed to be personally accountable for their contributions and to ensure that questions related to the accuracy or integrity of any part of the work, even ones in which the author was not personally involved, are appropriately investigated and resolved and the resolution documented in the literature. The corresponding author attests that all listed authors meet authorship criteria and that no others meeting the criteria have been omitted. SB and PS are the guarantors.

Funding: The study was funded by the Randers Regional Hospital Research Fund, Aarhus University, Health Research Fund of Central Denmark Region, Dagmar Marshall Foundation, Danish Regions Medical Foundation, Aase and Ejnar Danielsen Foundation, A.P. Moeller Foundation for the Advancement of Medical Science, and Soren Segels and Johanne Wiibroe Segels Research Fund. The funders had no role in the design of the study, the data collection, the data analysis, interpretation of data, or writing of the manuscript.

Competing interests: All authors have completed the ICMJE uniform disclosure form at www.icmje.org/coi_disclosure.pdf and declare: support for the submitted work as detailed above; no financial relationships with any organisations that might have an interest in the submitted work in the previous three years; no other relationships or activities that could appear to have influenced the submitted work.

Ethical approval: The CONDISOX trial was conducted in accordance with the ethical principles outlined in the latest version of the Declaration of Helsinki and the Guideline for Good Clinical Practice related to experiments on humans. The Central Denmark Region Committee on Biomedical Research Ethics and the Danish Health Authority approved the study before recruitment (EudraCT 2015 002942-30). The Medical Research Ethics Committees of the Amsterdam University Medical Centre approved the Dutch part of the study before recruitment. All participants gave informed written consent before taking part in the study.

Data sharing: Owing to Danish legislation, data will be available only after approval by the Danish Data Protection Agency and with a signed access agreement.

The lead authors (the manuscript's guarantors) affirm that this manuscript is an honest, accurate, and transparent account of the study being reported; that no important aspects of the study have been omitted; and that any discrepancies from the study as planned (and, if relevant, registered) have been explained.

Dissemination to participants and related patient and public communities: The results of the research will be disseminated to the participants and public through broadcasts, popular science articles, and newspapers.

Provenance and peer review: Not commissioned; externally peer reviewed.

This is an Open Access article distributed in accordance with the Creative Commons Attribution Non Commercial (CC BY-NC 4.0) license, which permits others to distribute, remix, adapt, build upon this work non-commercially, and license their derivative works on different terms, provided the original work is properly cited and the use is non commercial. See: http://creativecommons.org/licenses/by-nc/4.0/.

1 Martin JA, Hamilton BE, Osterman MJK, Driscoll AK. Births: Final Data for 2018. Natl Vital Stat Rep 2019;68:1-47.

2 World Health Organization. WHO recommendations: Induction of labour at or beyond term. 2018. https://www.ncbi.nlm.nih.gov/ pubmed/30629393.

3 Hedegaard M, Lidegaard $\varnothing$, Skovlund CW, Mørch LS, Hedegaard M. Reduction in stillbirths at term after new birth induction paradigm: results of a national intervention. BMJ Open 2014;4:e005785. doi:10.1136/bmjopen-2014-005785

4 Daly D, Minnie KCS, Blignaut A, et al. How much synthetic oxytocin is infused during labour? A review and analysis of regimens used in 12 countries. PLoS One 2020;15:e0227941. doi:10.1371/journal. pone.0227941

5 Simpson KR, Knox GE. Oxytocin as a high-alert medication implications for perinatal patient safety. MCN Am J Matern Child Nurs 2009;34:8-15, quiz 16-7. doi:10.1097/01. NMC.0000343859.62828.ee

6 Oláh KS, Steer PJ. The use and abuse of oxytocin. Obstet Gynaecol 2015;17:265-71. doi:10.1111/tog.12222

7 Mussi S, Incerti M, Plevani C, Ghidini A, Pezzullo JC, Locatelli A. Effect of oxytocin during labor on neonatal acidemia. J Matern Fetal Neonatal Med 2016;29:3098-103. doi:10.3109/14767058.2015.1114088

8 Tribe RM, Crawshaw SE, Seed P, Shennan AH, Baker PN. Pulsatile versus continuous administration of oxytocin for induction and augmentation of labor: two randomized controlled trials. Am J Obstet Gynecol 2012;206:230.e1-8. doi:10.1016/j.ajog.2011.11.001

9 Bostancı E, Kilicci C, Ozkaya E, Abide Yayla C, Eroglu M. Continuous oxytocin versus intermittent oxytocin for induction of labor: a randomized study. J Matern Fetal Neonatal Med 2020;33:651-6. doi: 10.1080/14767058.2018.1499092

10 Steer PJ, Carter MC, Choong K, Hanson M, Gordon AJ, Pradhan P. A multicentre prospective randomized controlled trial of induction of labour with an automatic closed-loop feedback controlled oxytocin infusion system. Br J Obstet Gynaecol 1985;92:1127-33. doi:10.1111/j.1471-0528.1985.tb03023.x

11 Budden A, Chen LJY, Henry A. High-dose versus low-dose oxytocin infusion regimens for induction of labour at term. Cochrane Database Syst Rev 2014;(10):CD009701. doi:10.1002/14651858. CD009701.pub2

12 Boie S, Glavind I, Velu AV, et al. Discontinuation of intravenous oxytocin in the active phase of induced labour. Cochrane Database Syst Rev 2018;8:CD012274. doi:10.1002/14651858.CD012274.pub2

13 Hernández-Martínez A, Arias-Arias A, Morandeira-Rivas A, Pascual-Pedreño Al, Ortiz-Molina EJ, Rodriguez-Almagro J. Oxytocin discontinuation after the active phase of induced labor: A systematic review. Women Birth 2019;32:112-8. doi:10.1016/j. wombi.2018.07.003

14 Vlachos D-EG, Pergialiotis V, Papantoniou N, Trompoukis S, Vlachos GD. Oxytocin discontinuation after the active phase of labor is established. / Matern Fetal Neonatal Med 2015:28:1421-7. doi:10.3 109/14767058.2014.955000

15 Saccone G, Ciardulli A, Baxter JK, et al. Discontinuing Oxytocin Infusion in the Active Phase of Labor: A Systematic Review and Meta-analysis. Obstet Gynecol 2017;130:1090-6. doi:10.1097/ AOG.0000000000002325

16 Eissaa AN, Sayyeda TM, El-Halabya AE, Tahoonb RA. The outcome of induced labor after oxytocin infusion discontinuation in the active phase. Menoufia Med J 2019;32:441-7. doi:10.4103/mmj. mmj_652_17

17 Mitra M, Sengupta M, Ghosal R, Saha C, Bandhya D, Ghosh D. Continuation versus Discontinuation of Oxytocin in Active Phase of Labour- A Randomised Trial. J Evol Med Dent Sci 2019;8:3342-6. doi:10.14260/jemds/2019/725

18 Boie S, Glavind I, Uldbjerg N, et al. CONDISOX-continued versus discontinued oxytocin stimulation of induced labour in a double-blind randomised controlled trial. BMC Pregnancy Childbirth 2019:19:320. doi:10.1186/s12884-019-2461-x

19 Marsál K, Persson PH, Larsen T, Lilja H, Selbing A, Sultan B. Intrauterine growth curves based on ultrasonically estimated foetal weights. Acta Paediatr 1996;85:843-8. doi:10.1111/j.1651-2227.1996.tb14164.x

20 Magann EF, Doherty DA, Chauhan SP, Busch FWJ, Mecacci F, Morrison JC. How well do the amniotic fluid index and single deepest pocket indices (below the 3rd and 5th and above the 95th and 97th percentiles) predict oligohydramnios and hydramnios? $A m$ J Obstet Gynecol 2004;190:164-9. doi:10.1016/S00029378(03)00859-7

21 Harris PA, Taylor R, Minor BL, et al, REDCap Consortium. The REDCap consortium: Building an international community of software platform partners. J Biomed Inform 2019;95:103208. doi:10.1016/j.jbi.2019.103208

22 Boie S, Lauridsen HH, Glavind J, Smed MK, Uldbjerg N, Bor P. The Childbirth Experience Questionnaire (CEQ)-Validation of its use in a Danish-speaking population of new mothers stimulated with oxytoci during labour. PLoS One 2020;15:e0233122. doi:10.1371/journal. pone.0233122

23 Bor P, Ledertoug S, Boie S, Knoblauch NO, Stornes I. Continuation versus discontinuation of oxytocin infusion during the active phase of labour: a randomised controlled trial. BJOG 2016;123:129-35. doi:10.1111/1471-0528.13589

24 Fayers PM, Machin D. Quality of Life: The Assessment, Analysis, and Interpretation of Patient-Reported Outcomes. 2nd ed. John Wiley \& Sons, 2013

25 Rooth G, Huch A, Huch R. Guidelines for the use of Fetal Monitoring. Int J Gynecol Obstet 1987;25:159-67.

26 Diven LC, Rochon ML, Gogle J, Eid S, Smulian JC, Quiñones JN. Oxytocin discontinuation during active labor in women who undergo labor induction. Am J Obstet Gynecol 2012;207:471.e1-8. doi:10.1016/j.ajog.2012.08.035

27 Ekelin M Svensson J, Evehammar S, Kvist LI Sense and sensibility: Swedish midwives' ambiguity to the use of synthetic oxytocin for labour augmentation. Midwifery 2015;31:e36-42. doi:10.1016/j. midw.2014.12.006

28 World Health Organization. WHO recommendations for induction of labour. WHO, 2011.

29 Dencker A, Berg M, Bergqvist L, Ladfors L, Thorsén LS, Lilja H. Early versus delayed oxytocin augmentation in nulliparous women with prolonged labour--a randomised controlled trial. BJOG 2009;116:530-6. doi:10.1111/j.1471-0528.2008.01962.x 